\title{
EMBALAGENS PLÁSTICAS E REFRIGERAÇÃO NA CONSERVAÇÃo PÓS-COLHEITA DE JABUTICABAS ${ }^{1}$
}

\author{
NICÁCIA PORTELLA MACHADO ${ }^{2}$, ENILTON FICK COUTINHO $^{3}$, ELISA RONDAN CAETANO $^{4}$
}

RESUMO - O objetivo deste trabalho foi avaliar a eficiência do uso de embalagens plásticas associadas à refrigeração na conservação pós-colheita de jabuticabas (Myrciaria spp) para consumo in natura e processamento. Realizaram-se dois experimentos, na Embrapa Clima Temperado - Pelotas-RS, com os seguintes tratamentos: $\mathrm{T}_{1}$ - testemunha (não-embaladas); $\mathrm{T}_{2}$ - bandeja plástica (capacidade da embalagem de $200 \mathrm{~g}$ ) (dimensões de $18 \mathrm{~cm}$ de comprimento x $12 \mathrm{~cm}$ de largura x $3 \mathrm{~cm}$ de altura) com duas perfurações de $1 \mathrm{~mm}$ cada, na tampa, e $\mathrm{T}_{3}$ - saco plástico (15 $\mu$ de espessura com perfurações de aproximadamente $0,5 \mathrm{~mm}$ ). No experimento 1 , os frutos (destinados ao consumo in natura) foram armazenados durante 08 dias, a $0^{\circ} \mathrm{C}$ e $90 \%$ UR, em câmara fria, com liberação de oxigênio ionizado $(0,03$ e $0,09 \mathrm{ppm}$ ) na atmosfera, mais dois dias a $20-22^{\circ} \mathrm{C}$ e $70-75 \%$ UR e, no experimento 2 (frutos destinados ao processamento), utilizou-se a mesma condição de armazenamento do experimento 1, porém as avaliações das jabuticabas foram realizadas 10 dias após o armazenamento a $0^{\circ} \mathrm{C}$ e $90 \%$ UR. Avaliaram-se a perda de peso (\%); sólidos solúveis totais (SST) ( $\left.{ }^{\circ} \mathrm{Brix}\right)$; $\mathrm{pH}$; acidez titulável (AT) $(\%$ de ácido cítrico) e relação SST/AT. Observou-se, em ambos os experimentos, que as jabuticabas embaladas com saco plástico de $15 \mu$ apresentaram significativamente menor perda de peso. A AT, SST, pH e relação SST/AT apresentaram variações entre os tratamentos, nos dois experimentos, sem afetar a qualidade comercial dos frutos. Conclui-se que o uso de saco plástico de $15 \mu$ de espessura e com perfurações de $0,5 \mathrm{~mm}$ é eficiente para manter a qualidade comercial de jabuticabas armazenadas durante 8 dias, a $0^{\circ} \mathrm{C}$ e $90 \%$ UR, mais dois dias a $20-22^{\circ} \mathrm{C}$ e $70-75 \%$ UR (frutos destinados ao consumo in natura) e por 10 dias a $0^{\circ} \mathrm{C}$ e $90 \%$ UR (frutos destinados ao processamento).

Termos para indexação: Myrciaria spp, qualidade de frutos, armazenamento refrigerado.

\section{PLASTIC PACKAGES AND COLD STORAGE ON THE POSTHARVEST PRESERVATION OF JABUTICABA FRUITS}

\begin{abstract}
The objective of this study was to evaluate the efficiency of the plastic packages use in cold storage on the preservation of jabuticabas (Myrciaria spp) for fresh consumption and processing. Two experiments were carried out at Embrapa Clima Temperado Research Center, Pelotas - RS, with the following treatments: $\mathrm{T}_{1}$ - control (open package); $\mathrm{T}_{2}-200 \mathrm{~g}$ capacity plastic box (dimensions: $18 \mathrm{~cm}$ of length, $12 \mathrm{~cm}$ of width and $3 \mathrm{~cm}$ of height) with perforated cover (two holes of $1 \mathrm{~mm}$ diameter each one); and $\mathrm{T}_{3}-15 \mu$ thick perforated plastic sac $(0,5 \mathrm{~mm}$ holes). In the Experiment 1 , the fruits (for fresh consume) with these packaging treatments were kept during eight days in a cold storage chamber under temperature of $0^{\circ} \mathrm{C}$ and $90 \%$ relative humidity (RH) containing $0,03-0,09 \mathrm{ppm}$ of ionized oxygen, and after being taken out from the cold storage, the fruits of the three pack kinds were left two days in room temperature $\left(20-22^{\circ} \mathrm{C}\right)$ and $70-75 \% \mathrm{RH}$. In the Experiment 2 (fruit for processing), it were evaluated the same treatments and cold storage conditions as it was referred in the first experiment, except that it was not simulated shelf life of the fruits which, in this experiment, were kept in the cold storage chamber of $0^{\circ} \mathrm{C}$ and $90^{\circ} \mathrm{C} \mathrm{RH}$ for 10 days, since the fruits were supposed to be used for processing. In both experiments, the variables evaluated were: loss of weight (\%); total soluble solids (TSS); $\mathrm{pH}$; tritable acidity -TA (\% citric acid); and ratio TSS/TA. It was concluded that for both purposes of fruit consumption the best packing was the $15 \mu$ plastic sac which kept better the quality during eight days in cold storage plus two days of simulated shelf life as well as the fruits for processing kept in cold storage for 10 days.
\end{abstract}

Index terms: Myrciaria spp, fruit quality, cold storage.

A jabuticabeira (Myrciaria spp.) é uma frutífera originada do Centro-Sul do Brasil, sendo cultivada do extremo sul ao extremo norte do País (Manica, 2000). As plantas produzem frutos com grande potencial tanto para consumo in natura como para a indústria (fabricação de geléia, compota, aguardente, licor, vinho e vinagre), porém tem como principal limitação a alta perecibilidade, reduzindo o período de armazenamento póscolheita e de comercialização (Donadio, 2000). Portanto, estudos sobre técnicas de armazenamento pós-colheita são necessários para prolongar o período de conservação sem afetar a qualidade das jabuticabas.

A atmosfera modificada é a técnica de armazenamento de frutos e hortaliças realizada sob condições de composição da atmosfera diferente daquela presente na atmosfera do ar normal (Lana \& Finger, 2000), alterando, principalmente, a concentração de gás carbônico $\left(\mathrm{CO}_{2}\right)$, oxigênio $\left(\mathrm{O}_{2}\right)$ e etileno (Pech et al., 1993).

\footnotetext{
(Trabalho 189-2005). Recebido em 23-11-2005. Aceito para publicação em 14-02-2007.

${ }^{2}$ Enga . Agríc, Doutoranda em Fruticultura de Clima Temperado - UFPEL, Pelotas/RS. nicacia@gmail.com

${ }_{3}^{3}$ Engenheiro Agrônomo, Dr. Pesquisador III, Embrapa Clima Temperado, Caixa Postal 403, CEP 96001-970, Pelotas/RS. enilton@cpact.embrapa.br

${ }^{4}$ Técnica agropecuária, Estagiária Embrapa Clima Temperado, Pelotas/RS nelisa@yahoo.com.br
} 
A modificação da atmosfera provoca decréscimo na velocidade da respiração, atraso na maturação e diminuição na deterioração de frutos e hortaliças (Kader et al., 1989), podendo ser obtida com o uso de materiais plásticos, como polietileno, policloreto de vinila e similares ou através da aplicação de produtos, como ceras, ésteres de sacarose e outros aditivos (Kluge et al., 2002).

O objetivo do trabalho foi avaliar a eficiência do uso de embalagens plásticas associadas à refrigeração na conservação pós-colheita de jabuticabas (Myrciaria spp) para consumo in natura ou processamento.

Foram realizados dois experimentos, em fevereiro de 2005, na Embrapa Clima Temperado, Pelotas-RS. Utilizaram-se jabuticabas maduras (Myrciaria spp.) colhidas de plantas localizadas na Estação Experimental de Cascata - Pelotas-RS. Após a colheita, realizou-se a seleção dos frutos de modo a uniformizar o tamanho dos mesmos (aproximadamente 18mm de diâmetro). Realizou-se caracterização inicial das jabuticabas a fim de verificar-se o estágio de maturação das mesmas. Para isso, verificou-se, em quatro repetições de 20 frutos, o peso médio dos frutos $(4,68 \mathrm{~g} /$ fruto), conteúdo de sólidos solúveis totais (SST) $\left(16,96^{\circ}\right.$ Brix $), \mathrm{pH}(3,58)$, acidez titulável (AT) $(0,58 \%$ de ácido cítrico) e relação SST/AT $(29,24)$.

Aos frutos maduros, em ambos os experimentos, foram aplicados os seguintes tratamentos: $\mathrm{T}_{1}$ - testemunha (nãoembaladas); $\mathrm{T}_{2}$ - bandeja plástica (capacidade da embalagem de $200 \mathrm{~g}$ ) (dimensões de $18 \mathrm{~cm}$ de comprimento $\mathrm{x} 12 \mathrm{~cm}$ de largura $\mathrm{x}$ $3 \mathrm{~cm}$ de altura) com duas perfurações de $1 \mathrm{~mm}$ cada, na tampa; $\mathrm{T}_{3}$, saco plástico $(15 \mu$ de espessura com perfurações de aproximadamente $0,5 \mathrm{~mm}$ ), sendo após submetidos a duas condições experimentais: no experimento 1 (frutos destinados ao consumo in natura), as jabuticabas foram armazenadas durante 08 dias, a $0^{\circ} \mathrm{C}$ e $90 \%$ UR, em câmara fria com liberação de oxigênio ionizado (concentração entre 0,03 e $0,09 \mathrm{ppm}$ ) na atmosfera, mais dois dias, a $20-22^{\circ} \mathrm{C}$ e $70-75 \%$ UR. O experimento 2 (frutos destinados ao processamento) constou da mesma condição de armazenamento descrito para o experimento 1, diferindo apenas quanto ao período de armazenamento (10 dias a $0^{\circ} \mathrm{C}$ e $90 \%$ UR). O oxigênio ionizado foi utilizado, em ambos os experimentos, apenas com a finalidade de diminuir a incidência de podridões pós-colheita.

Avaliaram-se as seguintes variáveis: a) perda de peso (expresso em percentagem (\%)); b) sólidos solúveis totais (SST) (expresso em ${ }^{\circ}$ Brix) por refratometria (Association Official Analytical Chemists - AOAC, 1992); c) pH; d) acidez titulável (AT) (\% ácido cítrico), conforme metodologia preconizada pelo Instituto Adolfo Lütz (1985) e; f) relação SST/AT.

O delineamento experimental utilizado foi o inteiramente casualizado, com quatro repetições de 20 frutos por tratamento (peso médio dos frutos de aproximadamente 5,0g). Após a análise da variância, as médias foram comparadas entre si, pelo teste de Duncan, a $5 \%$. Os dados percentuais originais foram transformados em arco-seno da raiz quadrada de $\mathrm{x} / 100$.

Nos dois experimentos (1 e 2), o uso de bandejas plásticas não foi eficiente na manutenção da qualidade dos frutos, tanto para consumo in natura como para processamento, devido à elevada perda de peso. Em ambos os experimentos, a perda de peso variou significativamente entre os tratamentos, sendo que as jabuticabas armazenadas sem embalagens (Testemunha) tiveram maior perda de peso (Tabelas 1 e 2). Brunini et al. (2004) verificaram que jabuticabas armazenadas sem o uso de filmes plásticos tiveram maior perda de peso após quatro dias de armazenamento a $11 \pm 1^{\circ} \mathrm{Ce} 98 \%$ UR e após dois dias em condições ambiente (23,6 a $28,3^{\circ} \mathrm{C} \mathrm{e} \mathrm{53,7} \mathrm{a} \mathrm{68,3 \%} \mathrm{UR).} \mathrm{Nos} \mathrm{experimentos} 1 \mathrm{e}$ 2 , o uso de saco plástico de $15 \mu$ reduziu significativamente a perda de peso (Tabelas 1 e 2). Segundo Gorris \& Peppelenbos (1992), a utilização de embalagens plásticas mantêm alta a umidade relativa ao redor do fruto, pois o déficit de pressão de vapor é menor no interior da embalagem, diminuindo a transpiração do fruto e, conseqüentemente, a perda de água.

Ao compararem-se os dois experimentos, observou-se que, em valores absolutos, as jabuticabas armazenadas somente sob refrigeração $\left(0^{\circ} \mathrm{C}\right.$ e $90 \%$ UR (experimento 2$\left.)\right)$ apresentaram, em todos os tratamentos, menor perda de peso (Tabela 2). Em relação ao Experimento 1, verificou-se que, com exceção das jabuticabas do tratamento $T_{3}$, as demais $\left(T_{1}\right.$ e $\left.T_{2}\right)$ desidrataram intensivamente (Tabela 1), apresentando aparência "enrugada", tornando-se impróprias para consumo in natura.

No experimento 1, as jabuticabas do tratamentotestemunha tiveram maiores valores de SST e de $\mathrm{pH}\left(16,38^{\circ}\right.$ Brix e 3,70 , respectivamente), porém diferindo estatisticamente apenas do tratamento $T_{3}$. A AT dos frutos do $T_{1}$ foi significativamente superior aos demais tratamentos $\left(\mathrm{T}_{2}\right.$ e $\left.\mathrm{T}_{3}\right)$, e a relação SST/AT não diferiu entre os mesmos (Tabela 1). No experimento 2, o SST foi significativamente menor no $\mathrm{T}_{3}$, e $\mathrm{o} \mathrm{T}_{2}$ apresentou menor AT e maior valor de $\mathrm{pH}$ e da relação SST/AT (Tabela 2). De acordo com Zagory \& Kader (1988), frutos armazenados com atmosfera modificada têm a atividade respiratória reduzida e, conseqüentemente, apresentam redução da conversão de amido em açúcares. Segundo Lima et al. (1996), a redução dos teores de $\mathrm{O}_{2}$ e o aumento de $\mathrm{CO}_{2}$, devido à modificação da atmosfera, reduzem a acidez durante o armazenamento refrigerado, causada pela redução da atividade enzimática relacionada ao metabolismo respiratório, elevando o pH dos frutos. A maior relação SST/AT verificada no tratamento $\mathrm{T}_{2}$ (bandejas plásticas) deve-se à variação do teor de SST e da AT.

Brunini et al. (2004), utilizando bandejas plásticas revestidas com filme plástico de PVC, no armazenamento refrigerado de jabuticabas 'Sabará', a $11^{\circ} \mathrm{C}$ e $98 \%$ UR, observaram redução da perda de peso e manutenção da aparência por até 6 dias, não influenciando, porém, nos teores de AT, SST e pH.

Durante a condução dos experimentos, não se observou incidência de podridões pós-colheita de frutos. Segundo Argenta et al. (2003), utilizando oxigênio ionizado na atmosfera de armazenamento, ocorre a produção de ozônio, que auxilia na redução da deterioração de frutos devido ao ataque de fungos, prolongando o período de conservação.

No presente trabalho, não foi determinada a concentração dos gases $\mathrm{O}_{2}$ e $\mathrm{CO}_{2}$ ao redor dos frutos, possibilitando, através dos resultados obtidos, apenas especular que as diferentes embalagens modificaram a atmosfera de armazenamento.

Saco plástico de $15 \mu$ de espessura e com perfurações de $0,5 \mathrm{~mm}$ é eficiente para manter a qualidade comercial de jabuticabas 
armazenadas durante 8 dias, a $0^{\circ} \mathrm{C}$ e $90 \%$ UR, mais dois, dias a 20 $22^{\circ} \mathrm{C}$ e $70-75 \%$ UR (frutos destinados ao consumo in natura) e por 10 dias, a $0^{\circ} \mathrm{C} \mathrm{e} 90 \% \mathrm{UR}$ (frutos destinados ao processamento).

TABELA 1 - Perda de Peso (\%), teores de sólidos solúveis totais (SST), pH, acidez titulável (AT) e relação SST/AT de jabuticabas após 8 dias sob refrigeração, a $0^{\circ} \mathrm{C}$ e $90 \%$ de UR, mais dois dias em ambiente de 20$22^{\circ} \mathrm{C}$ e $65-70 \%$ de UR. Embrapa Clima Temperado, Pelotas-RS, 2005.

\begin{tabular}{|c|c|c|c|c|c|}
\hline \multirow[b]{2}{*}{ Tratamentos } & \multicolumn{5}{|c|}{ Variáveis avaliadas } \\
\hline & $\begin{array}{c}\text { Perda de } \\
\text { peso } \\
(\%) \\
\end{array}$ & $\begin{array}{l}\text { SST } \\
\left({ }^{\circ} \text { Brix }\right)\end{array}$ & $\begin{array}{l}\text { AT } \\
\text { (\% ác. } \\
\text { cítrico) } \\
\end{array}$ & $\mathrm{pH}$ & SST/AT \\
\hline $\mathrm{T}_{1}$-Testemunha & $28,26 \mathrm{a}$ & $18,36 \mathrm{a}$ & $0,51 \mathrm{a}$ & $3,70 \mathrm{a}$ & $37,29 \mathrm{a}$ \\
\hline $\begin{array}{l}\mathrm{T}_{2} \text { - Bandeja } \\
\text { plástica }\end{array}$ & $19,34 \mathrm{~b}$ & $15,41 \mathrm{ab}$ & $0,41 \mathrm{~b}$ & $3,61 \mathrm{ab}$ & $35,92 \mathrm{a}$ \\
\hline $\begin{array}{l}\mathrm{T}_{3} \text { - Saco plástico } \\
(15 \mu)\end{array}$ & $3,49 \mathrm{c}$ & $13,21 \quad b$ & $0,43 \mathrm{~b}$ & $3,55 \mathrm{~b}$ & $30,53 \mathrm{a}$ \\
\hline CV $(\%)$ & 11,66 & 13,80 & 5,90 & 1,77 & 11,78 \\
\hline
\end{tabular}

Médias seguidas da mesma letra, na coluna, não diferem entre si, pelo teste de Duncan $(\mathrm{p}<0,05)$.

TABELA 2 - Perda de peso (\%), teores de sólidos solúveis totais (SST), pH, acidez titulável (AT) e relação SST/AT de jabuticabas após 10 dias sob refrigeração, a $0^{\circ} \mathrm{C}$ e $90 \%$ de UR. Embrapa Clima Temperado, Pelotas - RS, 2005.

\begin{tabular}{|c|c|c|c|c|c|}
\hline \multirow[b]{2}{*}{ Tratamentos } & \multicolumn{5}{|c|}{ Variáveis avaliadas } \\
\hline & $\begin{array}{l}\text { Perda de } \\
\text { peso }(\%)\end{array}$ & $\begin{array}{c}\text { SST } \\
\left({ }^{\circ} \text { Brix }\right)\end{array}$ & $\begin{array}{c}\text { AT } \\
\text { (\% ác. } \\
\text { cítrico) }\end{array}$ & $\mathrm{pH}$ & SST/AT \\
\hline $\mathrm{T}_{1}$-Testemunha & $10,24 \mathrm{a}$ & $16,11 \mathrm{a}$ & $0,37 \mathrm{a}$ & $3,57 \mathrm{~b}$ & $43,58 \mathrm{~b}$ \\
\hline $\begin{array}{l}\mathrm{T}_{2} \text { - Bandeja } \\
\text { plástica }\end{array}$ & $5,59 \mathrm{~b}$ & $15,46 \mathrm{a}$ & $0,28 \mathrm{c}$ & $3,75 \mathrm{a}$ & $55,81 \mathrm{a}$ \\
\hline $\begin{array}{l}T_{3} \text { - Saco plástico } \\
(15 \mu)\end{array}$ & $1,88 \mathrm{c}$ & $13,23 \mathrm{~b}$ & $0,32 \mathrm{~b}$ & $3,55 \mathrm{~b}$ & $40,68 \mathrm{~b}$ \\
\hline CV (\%) & 7,68 & 4,86 & 5,01 & 1,06 & 6,71 \\
\hline
\end{tabular}

Médias seguidas da mesma letra, na coluna, não diferem entre si, pelo teste de Duncan $(\mathrm{p}<0,05)$.

\section{REFERÊNCIAS}

ARGENTA, L.C.; BECKER, W.F.; KRAMMEF, J.G.; DEBOMA, E. Monitoramento da qualidade de pêssego armazenado sob atmosfera com oxigênio ionizado. Florianópolis: EPAGRI, 2003.11p.

ASSOCIATION OFFICIALANALYTICAL CHEMISTS. Official methods of analysis of the association of official analytical chemistry.11.ed. Washington, 1992.
BRUNINI, M. A.; OLIVEIRA, A. L. de; SALANDINI, C. A. R.; BAZZO, F. R. Influência de embalagens e temperatura no armazenamento de jabuticabas (Myrciaria jabuticaba (Vell) Berg) cv 'Sabará. Ciência e Tecnologia de Alimentos, Campinas, v.24, n.3, p.378-383, 2004.

DONADIO, L. C. Jabuticaba (Myrciaria jaboticaba (Vell.) Berg). Jaboticabal: FUNEP, 2000. 55p.

GORRIS, L. G. M.; PEPPELENBOS, H. W. Modified atmosphere and vacuum packaging to extend the shelf life of respiring food products. HortTechnology, Alexandria, v.2, n.3, p.303309, 1992.

INSTITUTO ADOLFO LUTZ. Normas técnicas do Instituto Adolfo Lütz: métodos químicos e físicos para análises de alimentos. São Paulo: EPU, 1985. 533p.

KADER, A. A.;ZAGORY, D.; KERBEL, E. L. Modified atmosphere packing of fruits and vegetables. Food Science and Nutrition, Hampshire, v.28, p.1-9, 1989.

KLUGE, R.A.; NATCHIGAL, J.C.; FACHINELLO, J.C.; BILHALVA, A. B. Fisiologia e manejo pós-colheita de frutas de clima temperado. Pelotas: UFPel, 2002. 214p.

LANA, M.M.; FINGER, F.L. Atmosfera modificada e controlada: aplicação na conservação de produtos hortícolas. Brasília: Embrapa Hortaliças, 2000.34p.

LIMA, L.C. de O.; SCALON, S. de P.Q.; SANTOS, J.E.S. Qualidade de mangas Mangifera indica) cv. 'Haden' embaladas com filme de PVC durante o armazenamento. Revista Brasileira de Fruticultura, Cruz das Almas, v.18, n.1, p.55-63, 1996.

MANICA, I. Frutas nativas, silvestres e exóticas 1: técnicas de produção e mercado: abiu, amora-preta, araçá, bacuri, biribá, carambola, cereja-do-rio-grande, jabuticaba. Porto Alegre: Cinco Continentes, 2000.327p.

PECH, J. C.; LATCHE, A.; BALAGUE, C. Cellular and molecular aspects of the plant hormone ethylene. Dordrecht: Kluwer Publisher, 1993.385p.

ZAGORY, D.; KADER, A.A. Modified atmosphere packing of fresh produce. Food Technology, Chicago, v.42, n.9, p.70-77, 1988. 